\title{
Our Focus Is on the Children
}

\author{
Rajasekharan P. Warrier, MD
}

Department of Pediatric Hematology/Oncology, Ochsner Clinic Foundation, New Orleans, LA; The University of Queensland School of Medicine, Ochsner Clinical School, New Orleans, LA; Department of Pediatrics, Division of Hematology/Oncology, Louisiana State University Health Sciences Center, New Orleans, LA

As guest editor of the winter issue of the Ochsner Journal, I am proud to present a special edition dedicated to children's issues. In fall 2012, I guest edited a theme issue of the Journal devoted to pediatric hematology/ oncology, but the current issue has a much broader focus, encompassing all of pediatrics and presenting a wide array of topics by contributors from many parts of the world.

The articles on nutrition and immune functions in sickle cell anemia by Kuvibidila et al and on advances in treatment of sickle cell disease by Gardner spotlight the significant amount of research being conducted in this field and the clear evidence of significant decreases in mortality and morbidity in children with the disease. Similarly, Kabir et al bring us up to date on advances in the use of immune checkpoint inhibitors in the treatment of pediatric cancers. Three papers on pediatric dentistry highlight the importance of the collaboration between pediatricians and the dental team in the management of complex dental disorders. Articles in the realm of social pediatrics explore children's perceptions of food security, pediatricians' promotion of smoking cessation among caregivers, the role of medical personnel in the prevention of child marriage in India, and the value of summer camps for children with sickle cell disease. From many different perspectives, these articles stress the need for pediatricians to be proactive in the global arena to prevent adverse mental, physical, and developmental effects on children. Both globally and domestically, famine and food insecurity, war and violence, and incarceration of parents and children secondary to illegal migration all have significant and profound effects on children. As medical personnel- and especially as pediatricians - it is important for us to have our voices heard for the kids' sakes.

Holt et al provide a thoughtful examination of how childhood deprivation of all kinds can lead to adult health consequences, and they give examples of successful programs developed to counter these negative childhood influences. Several unusual and clinically challenging case reports add variety and spice to this special issue and gave many of our residents and students the chance to contribute.

The Ochsner Department of Pediatrics has evolved over the years in clinical practice and in academic and clinical research and is now known as "Ochsner Hospital for Children." Our nationally ranked programs include pediatric cardiology and cardiovascular surgery as well as gastrointestinal and liver transplant. The Adolescent and Young Adult Clinic operated by a multidisciplinary team, including adult and pediatric oncologists, social workers, and mental health personnel, is unique and the only one in Louisiana. Multidisciplinary clinics for spina bifida and craniofacial and developmental disorders also offer critical and lifealtering care to patients and their families.

The children on the cover-one with sickle cell disease and one with cancer-have both had remarkable improvement in their conditions. These children are representatives of what we all work for-happy, healthy children.

My personal thanks to all the authors who contributed to this issue and to those who agreed to review the submissions. I hope this issue will act as an incentive for pediatricians here and across the world to conduct clinical research and inculcate the spirit of research and inquiry in their students and residents. 